\title{
Pathway Analysis for Plasma $\beta$-Amyloid, Tau and Neurofilament Light (ATN) in World Trade Center Responders at Midlife
}

\author{
Minos Kritikos - Sean A. P. Clouston - Erica D. Diminich • \\ Yael Deri - Xiaohua Yang - Melissa Carr - Sam Gandy · \\ Mary Sano · Evelyn J. Bromet · Benjamin J. Luft
}

Received: March 23, 2020 / Published online: April 30, 2020

(C) The Author(s) 2020

\section{ABSTRACT}

Introduction: World Trade Center (WTC) responders who aided in the search and rescue efforts are now at midlife, and evidence has

Digital Features To view digital features for this article go to https://doi.org/10.6084/m9.figshare.12145875.

M. Kritikos $(\bowtie)$. S. A. P. Clouston · E. D. Diminich Department of Family, Population and Preventive Medicine, Program in Public Health, Renaissance School of Medicine at Stony Brook, Stony Brook, NY, USA

e-mail: minos.kritikos@stonybrookmedicine.edu

Y. Deri $\cdot$ X. Yang

Department of Medicine, Renaissance School of Medicine at Stony Brook, Stony Brook, NY, USA

M. Carr · B. J. Luft

World Trade Center Health and Wellness Program, Department of Medicine, Renaissance School of Medicine at Stony Brook, Stony Brook, NY, USA

\section{S. Gandy}

Department of Neurology, Icahn School of Medicine at Mount Sinai, New York, NY, USA

S. Gandy · M. Sano

Department of Psychiatry, Icahn School of Medicine at Mount Sinai, NYC, New York, NY, USA

S. Gandy · M. Sano

James J Peters VAMC, Bronx, NY, USA

E. J. Bromet

Department of Psychiatry, Renaissance School of Medicine at Stony Brook, Stony Brook, NY, USA demonstrated that many are experiencing earlyonset cognitive impairment and are at risk of developing dementia, such as Alzheimer's disease (AD). According to the recent NIA-AA framework, $\mathrm{AD}$ is characterized by a neuropathological cascade commencing with $\beta$ amyloid deposition (A), followed by tauopathy $(\mathrm{T})$ and neurodegeneration $(\mathrm{N})$. However, the ATN model has not been replicated utilizing recently validated plasma-based biomarkers, and the role of the $A \beta_{40}$ subtype in $A$ is not well understood. This study examined plasma-based neuropathological markers of $A \beta_{42}$ and $A \beta_{40}$ for A, total tau for $\mathrm{T}$, and $\mathrm{N} f \mathrm{~L}$ for $\mathrm{N}$ in a cohort of World Trade Center responders at midlife in order to determine the role for the two $\beta$-amyloid subtypes in the ATN model.

Methods: Ultrasensitive Simoa technology was utilized to measure neuropathology in plasma collected from a consecutive clinical sample $(n=398)$. Generalized structural equation modeling was utilized for modeling linkages between pathological markers. Model fit was utilized to determine proposed directions of association.

Results: Our findings support the ATN neuropathological cascade model of $\mathrm{AD}$ and further identify an associative role for $A \beta_{40}$ in $A$ as playing a central role linking $\mathrm{T}$ to $\mathrm{N}$. A strong correlation was found between CI and age, and it was found that women may be at increased risk of elevated T levels, with plasma NfL levels higher in responders with CI. Notably, our 
model reported associations between: $A \beta_{42}$, CI and $\mathrm{N} ; \mathrm{A} \beta_{40}$, T and $\mathrm{N} ; \mathrm{T}$ and $\mathrm{CI} ; \mathrm{A} \beta_{42}$ and $\mathrm{A} \beta_{40}$. Conclusions: The current ATN model of AD does not specify the subtype of $\beta$-amyloid to be considered, which may be overlooking the differential roles that these two subtypes serve in the pathogenesis of $\mathrm{AD}$.

Keywords: Alzheimer's disease; ATN; $\beta$ Amyloid; Cognitive impairment; Dementia; Tau; Neurofilament light; Pathway analysis; Plasma; World Trade Center

\section{Key Summary Points}

\section{Why carry out this study?}

World Trade Center (WTC) responders are experiencing early-onset cognitive impairment and are at risk of developing dementia, such as Alzheimer's disease (AD).

This study examined the application of the NIA-AA framework for AD using Simoa plasma-based neuropathological markers of $A \beta_{42}$ and $A \beta_{40}$ for Amyloid, total tau for Tau and NfL for Neurodegeneration (ATN) in 398 WTC responders.

\section{What was learned from the study?}

Our findings support the ATN neuropathological cascade model of AD in WTC responders and identified a novel role for $A \beta_{40}$ in the ATN framework.

Plasma measures of ATN are a viable biomarker for assessing peripheral neuropathology underlying the cognitive impairment observed in WTC responders.

This study highlights that AD pathogenesis has different contributions from the each of the two $\beta$-amyloid subtypes, which the current ATN model does not specify.

\section{INTRODUCTION}

The World Trade Center (WTC) responders who aided in the search, rescue and recovery operations during and after the terrorist attacks of September 11, 2001, endured extraordinarily stressful experiences. Our work has demonstrated that these exposures resulted in high levels of chronic post-traumatic stress disorder (PTSD) in WTC responders that were associated with increased incidence of cognitive impairment (CI) [1-8], which can be prodromal to Alzheimer's disease (AD) [9-11].

$\mathrm{AD}$ is the sixth most common underlying cause of death [12]. AD has a long prodromal phase, with molecular, structural and functional changes commencing decades prior to the onset of observable symptoms. According to the recent National Institute on Aging-Alzheimer's Association (NIA-AA) framework [9-11], $\mathrm{AD}$ is characterized by progressive accumulation of an underlying neuropathological cascade commencing with $\beta$-amyloid deposition (A) [13], followed by tauopathy (T) [14] and neurodegeneration (N) [9], the extent and stage of which can be diagnosed by changes in $\mathrm{A}, \mathrm{T}$ and $\mathrm{N}$ (Fig. 1).

The preclinical phase of $\mathrm{AD}$ is characterized by cognitive decline and commences in midlife up to 20 years before clinical diagnosis $[15,16]$. However, whilst neuroimaging studies are propelling $\mathrm{AD}$ research forward [11], they are nevertheless limited in the number of ways that they can examine $A, T$ or $N$, given that such studies are costly and thus employ relatively small samples of older adults who are willing and able to undergo neuroimaging scans subject to radiation exposure from positron emission tomography (PET) radioligands. The recruitment of smaller and older age groups limits the collection and analysis of important information about critical time points for intervention. In addition, imaging studies are further limited by necessary exclusion criteria such as anxiety and/or claustrophobia, unsafe metal implants, or high body mass. Moreover, whilst imaging studies are able to identify A, T or N, they are unable to differentiate between the two main $A \beta$ subtypes $\left(A \beta_{40}\right.$ and $\left.A \beta_{42}\right)$ known to be 


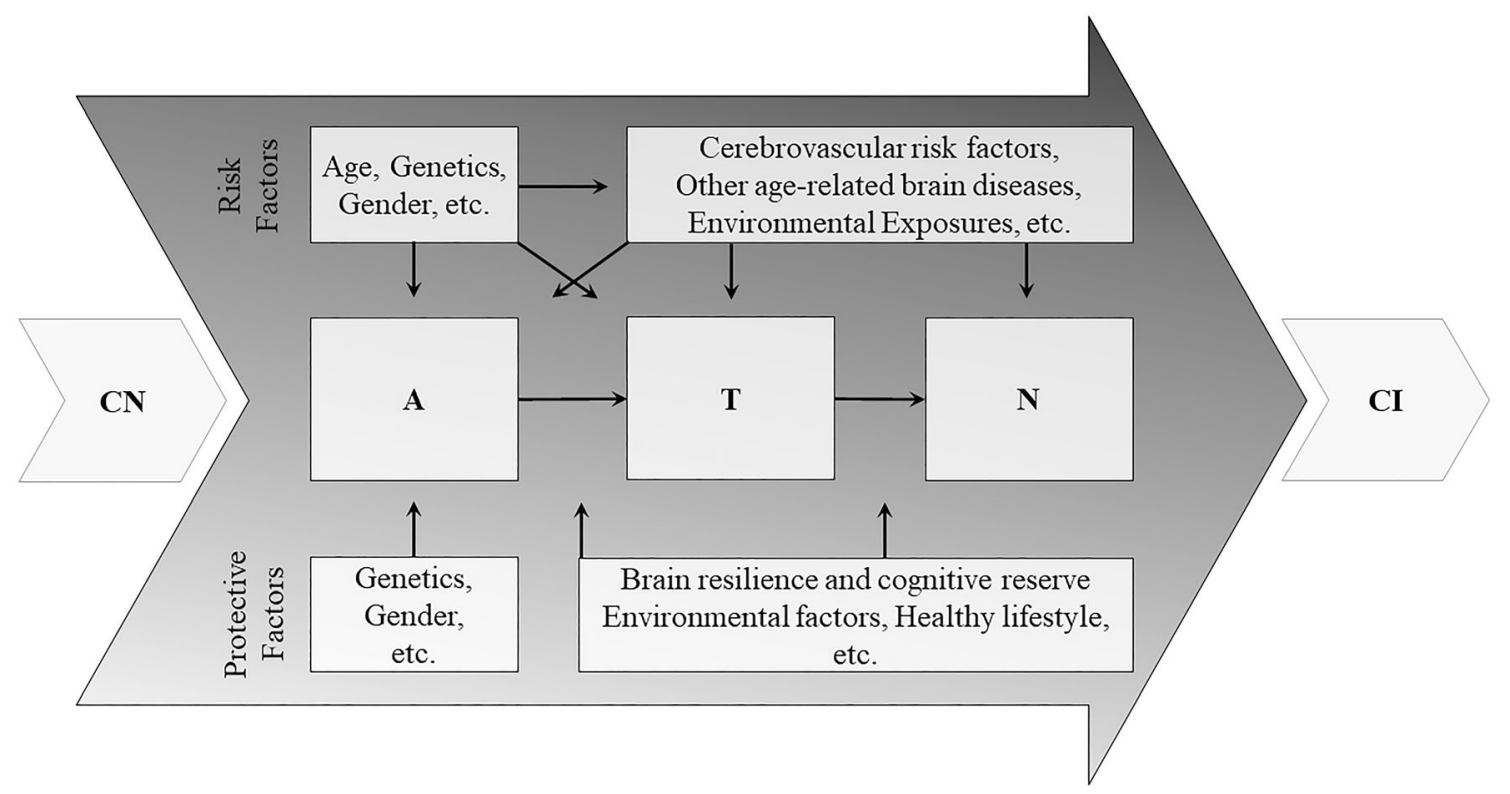

Fig. 1 Theoretical ATN research framework model showing a pathological cascade commencing with cognitively normal $(\mathrm{CN})$ and linking $\beta$-amyloid $(\mathrm{A})$, tau $(\mathrm{T})$ and neurodegeneration $(N)$ with cognitive impairment $(C I)$ in Alzheimer's disease, as well as the contributing relevant risk and protective factors. This model has been adapted from

implicated in AD. These important limitations highlight the need for implementing cost-effective and larger-sampled investigative modalities that can replicate imaging studies to better address the etiology and neuropathology depicted in the ATN model for AD.

As a solution to the above limitations, recent work supports the use of plasma-based measures for measuring $\mathrm{A}, \mathrm{T}$ and $\mathrm{N}$, utilizing protein distribution levels of $A \beta_{42}, A \beta_{40}[17,18]$, total tau for $\mathrm{T}[19,20]$ and neurofilament-light $(\mathrm{N} f \mathrm{~L})$ for $\mathrm{N}$ [21-24]. The main objective of this study was to determine the extent to which plasmabased measures for $\mathrm{A}_{42}, \mathrm{~A}_{40} \mathrm{~T}$ and $\mathrm{N}$ support the existing ATN model of AD neuropathological changes in a larger, age-heterogeneous World Trade Center (WTC) responder sample of men and women at midlife [25]. Additionally, since little is known about the role of $A \beta_{40}$ in $A$, a second aim of this effort was to further characterize the extent to which $A \beta_{40}$ is involved in the ATN model. the Sperling model [10] and updated to account for the NIA-AA Alzheimer's disease research framework $[9,11]$. $A \beta \beta$-amyloid, $T$ tauopathy, $N$ neurodegeneration

\section{METHODS}

\section{Setting}

Since 2002, the WTC Health Program (WTCHP) has monitored WTC responders. Eligibility for the program is overseen by the Centers for Disease Control and Prevention (CDC) [26]. These responders are now in their mid-50s, with the expected signs of senescence having already commenced. Considering the expected risks surrounding the aging process, prior work has noted that, compared with population norms, responders have a high prevalence of cognitive impairment at baseline [27] and incidence of mild cognitive impairment at follow-up [28]. Additionally, responders report greater memory dysfunction [7], and exhibit poor muscular strength [4], with an increased risk of physical functional limitations in neuro-associated movements [1]. 


\section{Ethics}

The present study received approval from the Stony Brook Institutional Review Board (IRB, CORIHS \#604113). All participants provided written informed consent, and this study was performed in accordance with the Helsinki Declaration of 1964 and its later amendments.

\section{Plasma Collection}

Blood plasma was acquired from 451 WTC responders in the first 5 months of 2019 during their annual cognitive monitoring protocol [8]. To control for sampling homogeneity, we excluded assays from 11 responders: eight due to the unsuccessful detection of certain neuropathological values, one for presenting with a history of stroke, and two with cognitive assessments that were deemed invalid. In total, the final analytical sample consisted of 398 responders whose background was viable and homogeneous for the purposes of this study. Age in years at time of blood draw and sex were also recorded.

Whole blood samples were collected in K2EDTA blood collection tubes (BD Vacutainer, Franklin Lakes, NJ) and subsequently placed on ice, followed by centrifugation at $2000 \mathrm{~g}$ for $15 \mathrm{~min}$ at $4{ }^{\circ} \mathrm{C}$. Plasma samples were then separated and subsequently placed in polyethylene tubes, prior to being stored at $-80^{\circ} \mathrm{C}$ pending transportation. Plasma samples were then transported in dry ice packages to be assayed at Quanterix $^{\mathrm{TM}}$ labs in Billerica, Massachusetts.

\section{Biomarker Measures}

We followed standard Quanterix ${ }^{\mathrm{TM}}$ protocols, whereby concentrations of neuropathological biomarkers in the plasma were analyzed by Simoa digital biomarker detection technology, a bead-based enzyme-linked immunosorbent assay for multiplex detection of proteins and nucleic acids at the lowest possible levels. The assay included monoclonal anti-A $\beta$ 40, anti$\mathrm{A} \beta_{42}$, anti-total tau and anti-N $f \mathrm{~L}$ antibodies, and lower limits of detection were set according to Quanterix protocols at 0.196, 0.045, 0.019 and
$0.104 \mathrm{pg} / \mathrm{ml}$, respectively. Samples were autodiluted at $4 \times$, and biomarker concentrations were calculated by utilizing data from two control subjects; none of our sampled assays presented below the lower limits of detection. In addition to the above biomarker measures, we considered that $\beta$-amyloid burden is increasingly being calculated by utilizing the $\mathrm{A} \beta_{42} / \mathrm{A} \beta_{40}$ ratio, where a lower ratio is believed to be more highly involved in the pathogenesis and neuropathology associated with AD [29]. However, for the present report, we further determined that co-adjustment for $A \beta_{42}$ and $A \beta_{40}$ in a statistical model is more efficient than just creating the ratio, and because our measures provide a stronger predictive model in line with prior pathological work, our analysis reports on both the $A \beta_{42} / A \beta_{40}$ ratio and on $A \beta_{42}$ and $A \beta_{40}$ as independent predictors.

\section{Cognitive Impairment Status}

The Montreal Cognitive Assessment (MoCA) is routinely utilized in the WTCHP to identify neurocognitive symptoms in the WTC cohort and can distinguish between cognitively normal $(\mathrm{CN})$ and CI with high specificity and sensitivity [30] by utilizing a cutoff score of $<23$ for CI $[31,32]$.

\section{Statistical Analysis}

Descriptive statistics for all demographic and clinical variables are provided, including variables such as MoCA scores and plasma levels for $\mathrm{A} \beta_{42}, \mathrm{~A} \beta_{40}$, $\mathrm{T}$ and $\mathrm{N}$, and the $\mathrm{A} \beta_{40} / \mathrm{A} \beta_{42}$ ratio, all of which were stratified by age groups, in addition to multiple individual one-way ordinary analysis of variance (ANOVA) with model fit tests to explore linear trends. We also stratified the responder sample into gender and then separately into $\mathrm{CI}$ versus $\mathrm{CN}$ status and applied multiple one-per-row unpaired $t$ test analyses. Multiple comparisons were controlled by false discovery rate determined by using a two-stage linear step-up procedure from Benjamini, Krieger and Yekutieli, with $Q=5 \%$. Each row was analyzed individually, without assuming a consistent SD. Results are reported as bar 
graphs, with error bars representing either standard deviation (SD), standard error of the mean (SEM), difference of means and standard error of the difference (SED), as noted in the text and figures.

We hypothesized that $\beta$-amyloid, tau and neurodegeneration neuropathology seen in $\mathrm{AD}$ and resulting cognitive impairment follows the ATN neuropathological cascade model. Therefore, we tested the predictive power of that model in these data using generalized structural equation modeling (GSEM) [33]. GSEM is unique in allowing the examination of associations between predictors and multiple outcomes in a flexible environment that permits the incorporation of outcomes that are not multivariate-normal. Notably, GSEM permits the analysis of count data that are right-skewed using log-linear Poisson regression [34] and furthermore allows for log-binomial models to then model multivariable-adjusted risk ratios in epidemiological data [35]. Results are reported graphically, and nonlinear assumptions were denoted using sub-textual boxes, along with estimated beta-coefficients (B), standard errors $(\mathrm{SE})$ and $p$ values $(p)$. Since GSEM does not allow for many of the model comparisons normally used with structural equation modeling (SEM), such as the root mean square error of approximation (RMSEA), model fit was evaluated by using both Akaike and Bayesian information criteria (AIC/BIC).

Because a central goal for this analysis was to test multiple pathological cascades, we utilized model fit to compare between possible neuropathological models by comparing model fit statistics to arrive at final models. In all models, demographic indicators (age/sex) were included if they were significantly associated with outcomes and model fit. Since existing models do not clarify the temporal linkages between $A \beta_{40}$ and $A \beta_{42}$, we relied on an assumption-free modeling approach. Specifically, all models incorporated $A \beta_{40}, A \beta_{42}, T$ and $\mathrm{N}$ as possible outcomes, and pathway directions were allowed to continue in either direction (i.e. $A \beta_{42} \rightarrow A \beta_{40}$, and the reverse), with the only condition being that bidirectional or cyclic associations were not allowed. Model fit also examined directionality, and $\Delta \mathrm{A} / \mathrm{BIC}$ was calculated to show the level of difference in model fit, noting that smaller A/BIC values indicate better fit. The best-fitting bidirectional pairings were included in the saturated model. Nonsignificant associations were excluded from the model sequentially, and the likelihood ratio test was used to compare the smaller model to the saturated model. The final model shown here includes all associations between predictors and outcomes that were deemed, using model fit, to be necessary to understand the underlying processes. Once the final model was identified, sensitivity analyses examined model fit by removing nonsignificant pathways. However, pathways were included, even if nonsignificant, if their removal reduced model fit. Finally, calculation of the predictive power of the derived model in predicting the risk of CI utilized the ratio of relative risks to compare the unadjusted risk ratio (RR) to the adjusted risk ratio (aRR). Analyses were completed using Stata 15/SE (StataCorp) and Prism 8 (Graphpad) software.

\section{RESULTS}

Sample characteristics are described in Table 1. Responders were predominantly male and on average at midlife when blood samples were collected. Approximately 13\% of responders in this sample had CI (Table 1). Stratification by age groups further revealed significant linear trends between each age group mean from left to right order for every variable under investigation (Fig. 2). Stratification by biological sex revealed that $\mathrm{T}(\mathrm{pg} / \mathrm{ml})$ was significantly higher in females $0.49 \quad(\mathrm{SE}=0.152)$ than males, $p=0.001$, which survived FDR (Fig. 3). Stratification by cognitive status revealed that responders with CI had (1) higher age (years): +3.09, $\operatorname{SED}=1.21, \quad p=0.011, q=0.011 ; \quad$ (2) lower $\mathrm{A} \beta_{42} \quad(\mathrm{pg} / \mathrm{ml}): \quad-1.08, \quad \mathrm{SED}=0.385$, $p=0.005, q=0.010$; (3) higher $\mathrm{A} \beta_{40}: \mathrm{A} \beta_{42}:+9$, SED $=3.26, \quad p=0.0175, \quad q=0.010 ; \quad$ and (4) higher $\mathrm{N}(\mathrm{pg} / \mathrm{ml}): \quad+1.53, \quad \mathrm{SED}=0.788$, $p=0.054, q=0.042$, all of which survived FDR set at $Q=5 \%$ (Fig. 4).

The AIC and BIC were minimized for the model commencing with $A \beta_{42}$ but flowing through $A \beta_{40}$ (Table 2). Results from the best- 
Table 1 Descriptive statistics for a WTC responder sample at midlife

\begin{tabular}{|c|c|c|c|c|c|c|c|}
\hline & $\begin{array}{l}\text { Age } \\
\text { (years) }\end{array}$ & $\begin{array}{l}\text { MoCA } \\
\text { (score) }\end{array}$ & $\begin{array}{l}\mathrm{A}_{42}(\mathrm{pg} / \\
\mathrm{ml})\end{array}$ & $\begin{array}{l}\mathbf{A} \beta_{40}(p g / \\
\mathrm{ml})\end{array}$ & $\begin{array}{l}\mathbf{A} \boldsymbol{\beta}_{40} / \mathbf{A \beta}_{42} \\
\text { (ratio) }\end{array}$ & $\begin{array}{l}\text { Total tau (pg/ } \\
\text { ml) }\end{array}$ & $\begin{array}{l}\mathrm{N} f \mathrm{~L}(\mathrm{pg} / \\
\mathrm{ml})\end{array}$ \\
\hline $25 \%$ Percentile & 49 & 24 & 8.01 & 196 & 20.7 & 1.18 & 6.61 \\
\hline Median & 54 & 26 & 9.63 & 219 & 22.6 & 1.61 & 8.54 \\
\hline 75\% Percentile & 59 & 27 & 11.2 & 247 & 26 & 2.05 & 11.7 \\
\hline Range & 43 & 15 & 19.9 & 335 & 434 & 4.09 & 32.5 \\
\hline Mean (SD) & $54.3(8.1)$ & $25.4(2.73)$ & $9.71(2.59)$ & $225(44.5)$ & $25.2(21.9)$ & $1.67(0.685)$ & $10(5.28)$ \\
\hline Sample size & $n=398$ & & & & & & \\
\hline $\begin{array}{l}\mathrm{CI} \\
\qquad(\mathrm{MoCA}<23)\end{array}$ & $n=51$ & $(12.81 \%)$ & & & & & \\
\hline Female & $n=21$ & $(5.28 \%)$ & & & & & \\
\hline
\end{tabular}

$S D$ standard deviation, $C I$ cognitive impairment, $p g / m l$ picograms per milliliter

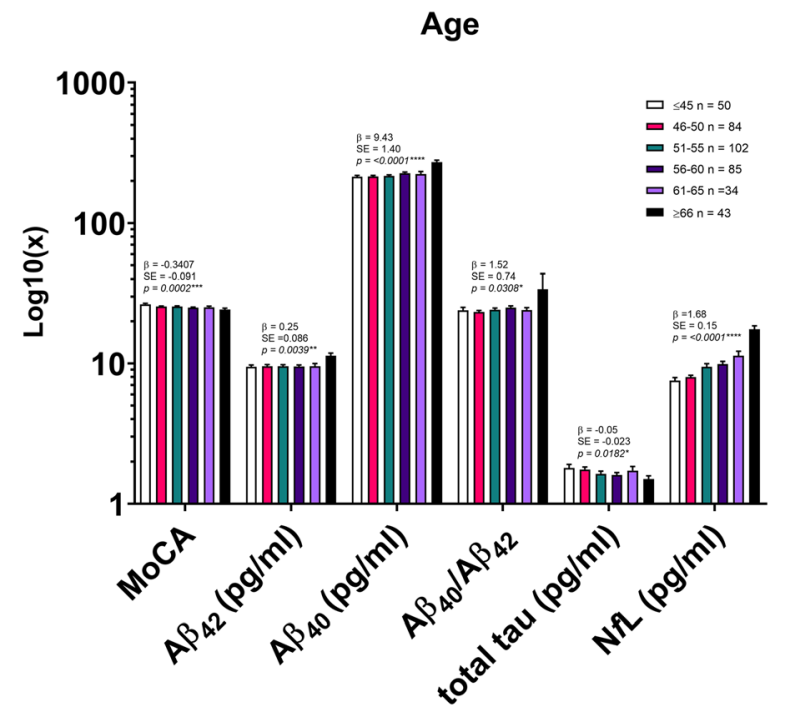

Fig. 2 Stratification by five age groups revealed significant linear trends between each age group mean from left to right order, as discovered by running an independent oneway ordinary ANOVA test for each variable. $\beta$ beta coefficient for slope, $S E$ standard error of slope. Data bars represent means, with error bars as the standard error of the mean. $Y$ axis values are $\log _{10}(x)$-transformed for comparative presentation

fitting model suggest that age played an independent role in predicting $A \beta_{40}, A \beta_{42}, T$ and $N$ distribution and that female responders had

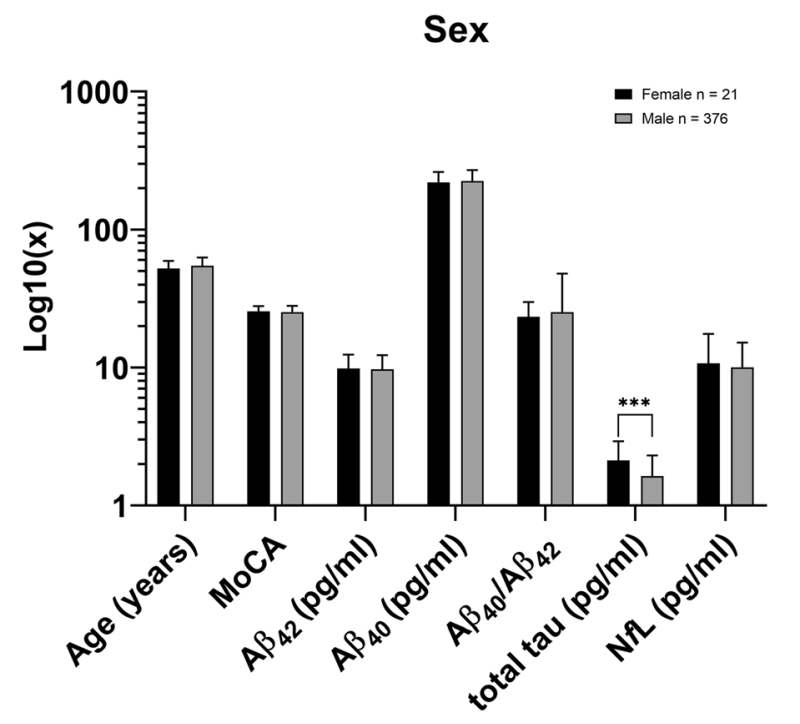

Fig. 3 Stratification by biological sex with multiple unpaired $t$ test analyses revealed a significantly higher difference in $\mathrm{T}$ pg/ml: $0.49(\mathrm{SED}=0.152)$ in females versus males, $p=0.001^{* * *}$, which survived FDR set at $Q=5 \%$. Data bars represent means, with error bars as standard deviations. SED standard error of the difference. $Y$ axis values are $\log _{10}(x)$-transformed for comparative presentation

higher levels of T. Our results support the proposed ATN framework view insofar as $\mathrm{A} \beta_{42} \rightarrow \mathrm{T} \rightarrow \mathrm{N}$; however, our findings suggest a 


\section{Cognitive Status}

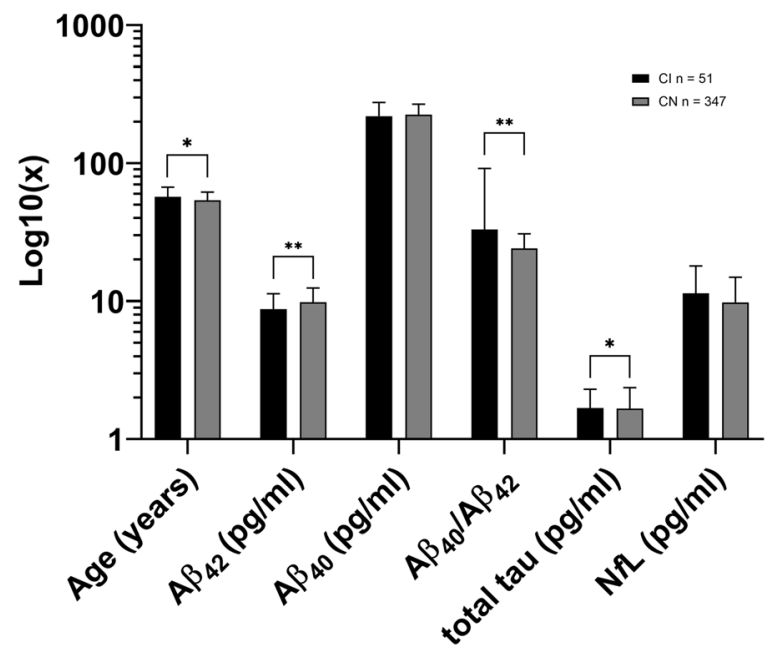

Fig. 4 Stratification by cognitive status (cognitively impaired [CI, MoCA $<23, n=51$ ] versus cognitively normal [CN, MoCA $>22, n=347])$. Multiple unpaired $t$ test analyses revealed that CI presented with significantly higher difference in age: $3.09, \operatorname{SED}=1.21, p=0.011$, $q=0.011$, significantly lower $\mathrm{A} \beta_{42} \mathrm{pg} / \mathrm{ml}:-1.08, \mathrm{SED}=$ $0.385, p=0.00, q=0.010$, significantly higher $\mathrm{A} \beta_{40} /$ $\mathrm{A} \beta_{42}$ ratio: $9, \mathrm{SED}=3.26, p=0.006, q=0.010$, and significantly higher $\mathrm{N} \mathrm{pg} / \mathrm{ml}: 1.53, \mathrm{SE}=0.788, p=0.054$, $q=0.042$, all of which survived FDR set at $Q=5 \%$. Data bars represent means, with error bars as standard deviations. $Y$ axis values are $\log _{10}(x)$-transformed for comparative presentation. $p g / m l$ picograms per milliliter, $C I$ cognitively impaired, $C N$ cognitively normal, $S E D$ standard error of difference

more intricate process indicating that $A \beta_{40}$ independently played a central, if late, role in linking $\mathrm{T}$ and $\mathrm{N}$ (Fig. 3). Examining the utility of demographic factors (age/sex), $\mathrm{A} \beta_{40}, \mathrm{~A} \beta_{42}$, $\mathrm{T}$ and $\mathrm{N}$ as predictors of $\mathrm{CI}$ in these data revealed that age, $A \beta_{42}$ and $T$ were independently associated with risk of CI (Fig. 3). Effect size calculations suggested that the risk of $\mathrm{CI}$ in responders increased by $30 \%$ per standard deviation (SD) reduction in $\mathrm{A} \beta_{42}(\mathrm{aRR}=1.30,[1.11-1.53])$, and by $24 \%$ per $\mathrm{SD}$ increase in $\mathrm{T}(\mathrm{aRR}=1.24$ [1.05-1.49]). The likelihood ratio test showed no loss of information in the model presented here as compared with the saturated model $\left(\chi_{9}^{2}=-9.90, p=1.000\right)$ (Fig. 5).

\section{DISCUSSION}

In this study, we examined the $\mathrm{AD}$ pathological ATN framework cascade utilizing novel highsensitivity plasma-based measures of neuropathology collected in a sample of WTC responders. Demographic correlations were interesting, as CI strongly associated with age, but more so that women may be at increased risk of $\mathrm{T}$. There is currently sparse prior work to suggest that sex should act as a predictive element for the distribution and accumulation of tau, other than a neuroimaging study showing elevated $\mathrm{T}$ in female APOE4 carriers [36].

An important goal of this study was to disentangle how the two $\beta$-amyloid subtypes, $A \beta_{42}$ and $A \beta_{40}$, flow through the ATN model; thus we examined a more refined $A \beta_{40}, A \beta_{42}, T$ and $N$ cascade model of AD. Our findings provide strong support for the $\mathrm{A} \beta_{42} \rightarrow \mathrm{T} \rightarrow \mathrm{N}$ model, with biological sex differences indicating that the pathogenesis of $\mathrm{T}$ differs among female responders and that $A \beta_{42}$ is associated with an increased risk of $\mathrm{CI}$, which supports the translocation theory of $\mathrm{A}_{42}$ from the periphery into the brain [37], as responders with CI had lower raw mean plasma levels. However, we also report that $A \beta_{40}$ offers an intermediary role linking the proliferation of $\mathrm{T}$ with changes in $\mathrm{N}$, which suggests that different types of amyloid may play different roles in the progression of AD. We further report an elevated $A \beta_{40}: A \beta_{42}$ ratio in responders with $\mathrm{CI}$, which is in agreement with previous reports for individuals at risk for $\mathrm{AD}[29,37,38]$, which is in agreement with previous observations for $\mathrm{AD}$ pathogenesis [21].

Prior studies have noted that $\mathrm{T}$ incompletely follows A [25], and we suggest that one reason for this mismatch is due to $\beta$-amyloid subtypes $\left(A \beta_{40}\right.$ and $\left.A \beta_{42}\right)$ differentially regulating the course of $\mathrm{T}$ and $\mathrm{N}$ neuropathology. Notably, our responder sample demonstrated associations for $\mathrm{A} \beta_{42}$ with $\mathrm{CI}$ and $\mathrm{N}, \mathrm{A} \beta_{40}$ with $\mathrm{T}$ and $\mathrm{N}$, $\mathrm{T}$ with $C I$, and $A \beta_{42}$ with $A \beta_{40}$. The fact that $A \beta_{42}$ and $\mathrm{A} \beta_{40}$ have these differential roles in the ATN model underscores a possible explanation for the previously reported mismatches between A and $\mathrm{T}$ seen in imaging studies of $\mathrm{AD}$. Indeed, 
Table 2 Model fit statistics to determine pathway analysis model selection

\begin{tabular}{lllll}
\hline Pathway & $\begin{array}{l}\text { Akaike } \\
\text { information } \\
\text { criteria }\end{array}$ & $\begin{array}{l}\text { Bayesian } \\
\text { information } \\
\text { criteria }\end{array}$ & $\begin{array}{l}\text { Improvement in model fit compared to } \\
\text { demographically adjusted base model }\end{array}$ & $\begin{array}{l}\text { Change in } \\
\text { fit (2-1) }\end{array}$ \\
\hline$(1) \quad \mathrm{A} \beta_{42} \rightarrow \mathrm{A} \beta_{40}$ & $\mathbf{1 0 , 3 3 9 . 2 5}$ & $\mathbf{1 0 , 3 8 8 . 2 4}$ & -180.37 & 19.62 \\
$(2) \quad \mathrm{A} \beta_{40} \rightarrow \mathrm{A} \beta_{42}$ & $10,358.87$ & $10,407.86$ & -160.75 & \\
$(1) \mathrm{A} \beta_{42} \rightarrow \mathrm{T}$ & $\mathbf{1 0 , 5 1 1 . 5}$ & $\mathbf{1 0 , 5 6 0 . 4 9}$ & -8.12 & 6.03 \\
$(2) \mathrm{T} \rightarrow \mathrm{A} \beta_{42}$ & $10,517.53$ & $10,566.52$ & -2.09 & \\
$(1) \mathrm{A} \beta_{42} \rightarrow \mathrm{N}$ & $\mathbf{1 0 , 4 7 5 . 1 8}$ & $\mathbf{1 0 , 5 2 4 . 1 6}$ & -44.44 & 33.23 \\
$(2) \mathrm{N} \rightarrow \mathrm{A} \beta_{42}$ & $10,508.41$ & $10,557.4$ & -11.21 & \\
$(1) \mathrm{A} \beta_{40} \rightarrow \mathrm{T}$ & $10,518.64$ & $10,567.63$ & -0.98 & -9.49 \\
$(2) \mathrm{T} \rightarrow \mathrm{A} \beta_{40}$ & $\mathbf{1 0 , 5 0 9 . 1 5}$ & $\mathbf{1 0 , 5 5 8 . 1 3}$ & -10.47 & 29.41 \\
$(1) \mathrm{A} \beta_{40} \rightarrow \mathrm{N}$ & $\mathbf{1 0 , 4 6 7 . 9 5}$ & $\mathbf{1 0 , 5 1 6 . 9 3}$ & -51.67 & \\
$(2) \mathrm{N} \rightarrow \mathrm{A} \beta_{40}$ & $10,497.36$ & $10,546.35$ & -22.26 & 1.67 \\
$(1) \mathrm{T} \rightarrow \mathrm{N}$ & $\mathbf{1 0 , 5 1 9 . 8 5}$ & $\mathbf{1 0 , 5 6 8 . 8 3}$ & 0.23 & \\
$(2) \mathrm{N} \rightarrow \mathrm{T}$ & $10,521.52$ & $10,570.51$ & 1.9 & \\
\hline
\end{tabular}

The smallest Akaike/Bayesian information criteria, denoted in bold, indicate the best-fitting directional pathway. All models adjusted for associations between age and all outcomes

from studies of histopathology, we see that $A \beta_{40}$ plays a critical role in modulating dense-core plaque formation, which is critical in $\mathrm{AD}$ pathogenesis [39], and $A \beta_{42}$, more commonly present in diffuse plaques [40], are often collocated with $\mathrm{T}$ [41], and more commonly evident in the occipital lobe [42] in later stages of sporadic AD [43]. Furthermore, in neuroimaging studies, we see dense-core $A \beta_{40}$ plaques that tend to be smaller but denser than $A \beta_{42}$ diffuse plaques [42]. Therefore, the two subtypes of $\beta$ amyloid, though highly inter-related, could result in both $\mathrm{A} \beta_{42}$-related cognitive changes, with a potentially slower onset of CI via changes in $T$ through $A \beta_{40}$ and $N$. This further highlights the temporal elements underscoring the ATN model, where in certain cases $\mathrm{A} \beta_{40}$ may be more prominent in leading towards $\mathrm{T}$ and $\mathrm{N}$, in the absence of pronounced $A \beta_{42}$ accumulation. Indeed, studies have demonstrated inverse associations between plasma $A \beta_{42} / \mathrm{A} \beta_{40}$ ratios and high cortical A burden [29], which taken together with our report highlights the role for
$\mathrm{A} \beta_{40}$ in the ATN model. Interestingly, prior work from our lab demonstrated that in WTC responders with PTSD, $A \beta_{42} / A \beta_{40}$ ratios were higher [44], suggesting a leading elevation in $\mathrm{A} \beta_{42}$ when applying the ATN model to WTCPTSD.

\section{STRENGTHS AND LIMITATIONS}

This is the first study to apply the ATN research framework using an ultrasensitive multiplex array of plasma-based neuropathological biomarkers in a large, well-characterized sample of individuals at midlife-the WTC responder cohort. In addition, while prior imaging studies are able to identify ATN, they lack specificity in their ability to differentiate between $A \beta_{40}$ and $\mathrm{A} \beta_{42}$, which this study accomplishes. Furthermore, the GSEM analytical approach used to determine and examine plasma ATN in WTC responders allows for examination of associations of predictors with non-normal 


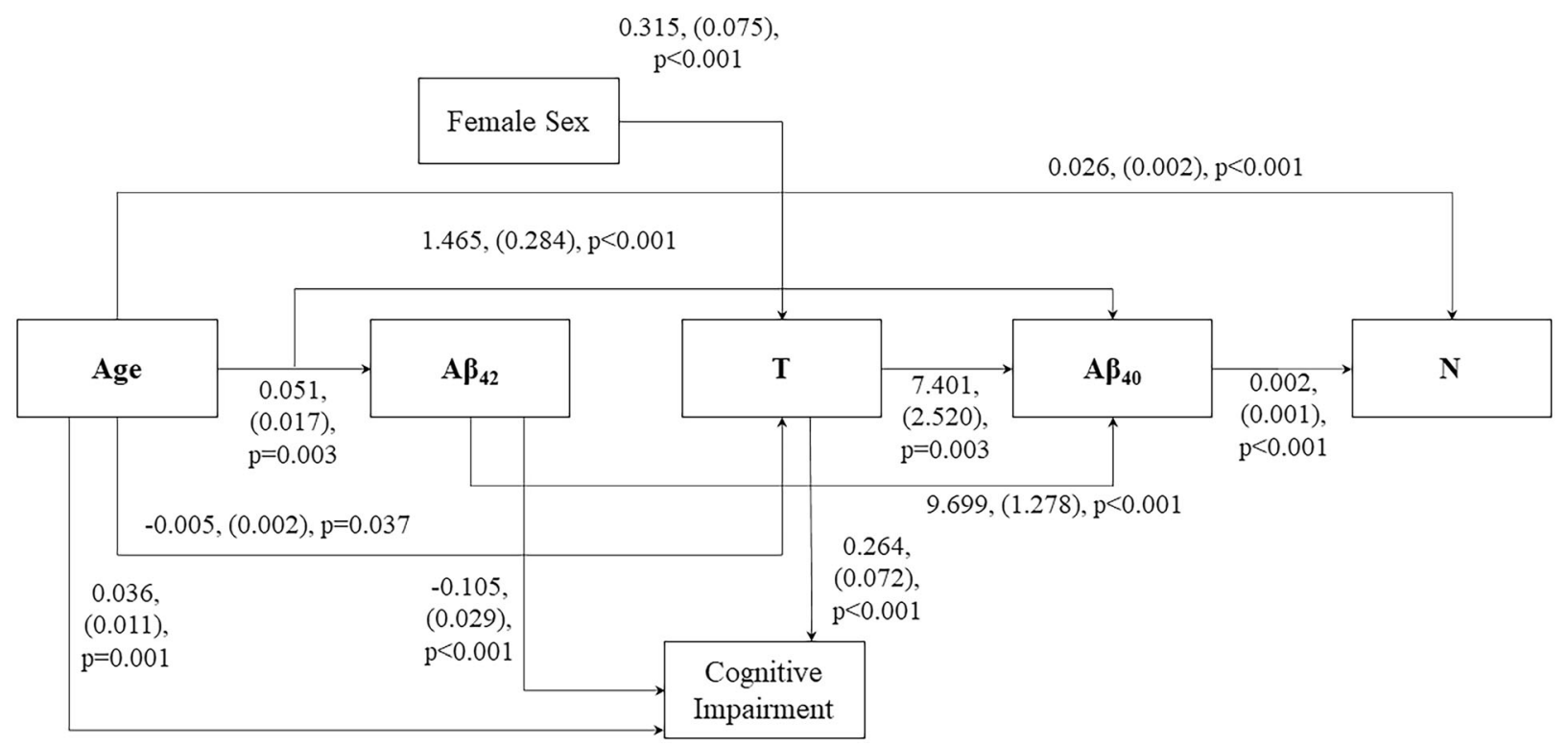

Fig. 5 Pathway analysis examining the linkages between demographic and neuropathological features and risk of cognitive impairment in World Trade Center responders. Results expressed as unstandardized beta coefficients, (standard error), $p$ values. Only significant results are shown. The likelihood ratio test showed no loss of

distributions, thus enabling us to fully characterize the peripheral distribution of $A \beta_{40}, A \beta_{42}$, $\mathrm{T}$ and $\mathrm{N}$.

Nevertheless, this study lacked an external control group with which to compare peripheral levels of ATN. Since the present report was an initial and exploratory application of the ATN research framework for these blood-based biomarkers in a population of WTC responders with a spectrum of cognitive impairment, we propose that future such investigations with this cohort employ an external control group in order for the findings to be more generalizable. Furthermore, this study focused on an occupational population of adults exposed to the unique events of the WTC attacks and the chronic aftermath of recovery efforts within New York City, a geographic region where responders also resided. Though the use of GSEM represents a notable strength of this study, it nevertheless does not permit the calculation of a fit statistic (RMSEA) that is commonly employed in SEM. Finally, we measured plasma total tau as a marker for $\mathrm{T}$ and not information in the model presented here as compared with the saturated model $\left(\chi_{9}^{2}=-9.90, p=1.000\right)$. $A \beta \beta$ amyloid, $T$ total tau, $N$ neurofilament-light

plasma phospho-tau181. Whilst both have been demonstrated to effectively distinguish between $\mathrm{AD}$ and cognitively normal subjects in a clinical setting, pTau181 has been reported to be a more sensitive and specific predictor of elevated brain $\mathrm{A} \beta$ and was associated with tau PET, whereas total tau was associated with cortical thickness in $\mathrm{AD}$ signature regions [45], which is an association that applies to our finding of reduced cortical thickness in the WTC responder population [46]. Therefore, future pathway analyses and considerations of the ATN model in AD pathogenesis should not only account for both subtypes of $\beta$-amyloid, but should also take into account both isoforms of plasma tau. These factors limit the generalizability of the findings in our study. However, prior studies in our group with the WTC responder population have provided extensive characterizations for the role of neuro-behavioral symptoms of PTSD and major depressive disorder (MDD) and their associations with CI [2, 6, 8, 28, 47]. Therefore, we encourage future studies that aim to examine the neurobiology of such neurobehavioral 
disorders in WTC responders to employ the ATN research framework and novel modeling approaches we report here.

\section{CONCLUSIONS}

The current ATN model of AD does not specify the subtype of $\beta$-amyloid to be considered. Here, we demonstrate that $A \beta_{40}$ levels are important indicators for $\mathrm{T}$ and $\mathrm{N}$, and although $A \beta_{40}$ and $A \beta_{42}$ were strongly interrelated, they do not co-occur uniformly, such that some individuals may develop $A \beta_{42}$ but not $A \beta_{40}$, or the inverse. If so, then there may be adults who appear $\mathrm{A}+$ on a neuroimaging scan but never develop $A \beta_{40}$ dense-core plaques. Conversely, some individuals with rapid $A \beta_{40}$ may not attain the levels of global $\beta$-amyloid deposition necessary to be considered $\mathrm{A}+$, potentially earning the diagnosis of primary age-related tauopathy (PART) [48] or suspected non-Alzheimer's disease pathophysiology (SNAP) [49]. Therefore, the present study suggests that when considering the current ATN model of AD in a clinical setting, we may be overlooking the differential roles that the two $\beta$-amyloid subtypes play in the pathological cascade. Furthermore, the present report highlights the applicability and utility of employing analysis of peripheral ATN biomarkers to study and further understand the underlying neurobiological etiology for the CI observed in this population. Combined with molecular imaging methodologies that directly interrogate levels of cortical ATN, future investigations utilizing a concurrent imaging and peripheral ATN investigation offer a powerful arsenal of useful biomarkers to help better understand the observed $\mathrm{CI}$ in this population.

\section{ACKNOWLEDGEMENTS}

The authors would like to acknowledge the support from the National Institute for Occupational Safety and Health (NIOSH), who support World Trade Center clinical and monitoring efforts at Stony Brook University (CDC/NIOSH 200-2011-39361). Finally, we would like to kindly thank all WTC responders who participated in this study.

Funding. We would also like to acknowledge the support for aging-related research in this population (NIH/NIA R01 049953), and for funding to support brain aging research and treatment (NIH/NIA P50 AG005138).

Authorship. All named authors meet the International Committee of Medical Journal Editors (ICMJE) criteria for authorship for this article, take responsibility for the integrity of the work as a whole, and have given their approval for this version to be published.

Disclosures. Minos Kritikos, Sean A. P. Clouston, Erica D. Diminich, Yael Deri, Xiaohua Yang, Melissa Carr, Sam Gandy, Mary Sano, Evelyn J. Bromet and Benjamin J. Luft have nothing to disclose.

Compliance with Ethics Guidelines. The present study received approval from the Stony Brook Institutional Review Board (IRB, CORIHS \#604113). All participants provided written informed consent, and the study was performed in accordance with the Helsinki Declaration of 1964 and its later amendments.

Data Availability. The datasets generated during and/or analyzed during the current study are available from the corresponding author on reasonable request.

Open Access. This article is licensed under a Creative Commons Attribution-NonCommercial 4.0 International License, which permits any non-commercial use, sharing, adaptation, distribution and reproduction in any medium or format, as long as you give appropriate credit to the original author(s) and the source, provide a link to the Creative Commons licence, and indicate if changes were made. The images or other third party material in this article are included in the article's Creative Commons licence, unless indicated otherwise in a credit line to the material. If material is not included in the article's Creative Commons licence and your intended use is not permitted by statutory 
regulation or exceeds the permitted use, you will need to obtain permission directly from the copyright holder. To view a copy of this licence, visit http://creativecommons.org/licenses/by$\mathrm{nc} / 4.0 /$.

\section{REFERENCES}

1. Clouston SAP, Guralnik JM, Kotov R, Bromet EJ, Luft BJ. Functional limitations among responders to the world trade center attacks 14 years after the disaster: implications of chronic posttraumatic stress disorder. J Trauma Stress. 2017;30(5):443-52.

2. Clouston SA, Kotov R, Pietrzak RH, Luft BJ, Gonzalez A, Richards M, et al. Cognitive impairment among World Trade Center responders: long-term implications of re-experiencing the 9/11 terrorist attacks. Alzheimers Dement (Amst). 2016;2016(4): 67-75.

3. Clouston SAP, Shapira O, Kotov R, Lei L, Waszczuk $\mathrm{M}$, Bromet EJ, et al. Proton pump inhibitors and the risk of severe cognitive impairment: the role of posttraumatic stress disorder. Alzheimers Dement (N Y). 2017;3(4):579-83.

4. Mukherjee S, Clouston S, Kotov R, Bromet E, Luft B. Handgrip strength of World Trade Center (WTC) responders: the role of re-experiencing posttraumatic stress disorder (PTSD) symptoms. Int J Environ Res Public Health. 2019;16(7):1128.

5. Galea S, Ahern J, Resnick H, Kilpatrick D, Bucuvalas M, Gold J, et al. Psychological sequelae of the September 11 terrorist attacks in New York City. N Engl J Med. 2002;346(13):982-7.

6. Clouston S, Deri Y, Diminich E, Kew RR, Kotov R, Stewart C, et al. Posttraumatic stress disorder associated with total amyloid burden and amyloid- $\beta$ 42/40 ratios in plasma: results from a pilot study of World Trade Center responders. Alzheimer's Dement. 2019;11:216-20.

7. Clouston S, Pietrzak RH, Kotov R, Richards M, Spiro A 3rd, Scott S, et al. Traumatic exposures, posttraumatic stress disorder, and cognitive functioning in World Trade Center responders. Alzheimers Dement (N Y). 2017;3(4):593-602.

8. Clouston S, Kotov R, Pietrzak R, Richards M, Spiro III A, Mukherjee $S$, et al. Excess incidence of mild cognitive impairment in World Trade Center responders: evidence from a longitudinal study of cognitive functioning. Manuscript submitted for publication (copy on file with author).
9. Knopman DS, Haeberlein SB, Carrillo MC, Hendrix JA, Kerchner G, Margolin R, et al. The National Institute on Aging and the Alzheimer's Association Research Framework for Alzheimer's disease: perspectives from the research roundtable. Alzheimers Dement. 2018;14(4):563-75.

10. Sperling RA, Aisen PS, Beckett LA, Bennett DA, Craft $S$, Fagan AM, et al. Toward defining the preclinical stages of Alzheimer's disease: recommendations from the National Institute on Aging-Alzheimer's Association workgroups on diagnostic guidelines for Alzheimer's disease. Alzheimers Dement. 2011;7(3):280-92.

11. Jack CR Jr, Bennett DA, Blennow K, Carrillo MC, Dunn B, Haeberlein SB, et al. NIA-AA research framework: toward a biological definition of Alzheimer's disease. Alzheimers Dement. 2018;14(4): 535-62.

12. Alzheimer's Association. 2018 Alzheimer's disease facts and figures. Alzheimer's Dement. 2018;14(3): 367-429.

13. Vemuri P, Lesnick TG, Przybelski SA, Graff-Radford J, Reid RI, Lowe VJ, et al. Development of a cerebrovascular magnetic resonance imaging biomarker for cognitive aging. Ann Neurol. 2018;84(5): 705-16.

14. Maass A, Lockhart SN, Harrison TM, Bell RK, Mellinger $\mathrm{T}$, Swinnerton $\mathrm{K}$, et al. Entorhinal tau pathology, episodic memory decline, and neurodegeneration in aging. J Neurosci. 2018;38(3): 530-43.

15. Singh-Manoux A, Kivimaki M, Glymour MM, Elbaz A, Berr C, Ebmeier KP, et al. Timing of onset of cognitive decline: results from Whitehall II prospective cohort study. BMJ. 2012;344:d7622.

16. Nahhas RW, Choh AC, Lee M, Chumlea WM, Duren DL, Siervogel RM, et al. Bayesian longitudinal plateau model of adult grip strength. Am J Hum Biol. 2010;22(5):648-56.

17. Nakamura A, Kaneko N, Villemagne VL, Kato T, Doecke J, Dore V, et al. High performance plasma amyloid-beta biomarkers for Alzheimer's disease. Nature. 2018;554(7691):249-54.

18. Deane R, Bell RD, Sagare A, Zlokovic BV. Clearance of amyloid-beta peptide across the blood-brain barrier: implication for therapies in Alzheimer's disease. CNS Neurol Disord Drug Targets. 2009;8(1): 16-30.

19. Noble W, Hanger DP, Miller CC, Lovestone S. The importance of tau phosphorylation for neurodegenerative diseases. Front Neurol. 2013;4:83. 
20. Mattsson N, Zetterberg H, Janelidze S, Insel PS, Andreasson U, Stomrud E, et al. Plasma tau in Alzheimer disease. Neurology. 2016;87(17):1827-35.

21. Mattsson N, Cullen NC, Andreasson U, Zetterberg $\mathrm{H}$, Blennow K. Association between longitudinal plasma neurofilament light and neurodegeneration in patients with Alzheimer disease. JAMA Neurol. 2019;76(7):791-9.

22. Steinacker P, Semler E, Anderl-Straub S, Diehl-Schmid J, Schroeter ML, Uttner I, et al. Neurofilament as a blood marker for diagnosis and monitoring of primary progressive aphasias. Neurology. 2017;88(10):961-9.

23. Kuhle J, Barro C, Andreasson U, Derfuss T, Lindberg $\mathrm{R}$, Sandelius A, et al. Comparison of three analytical platforms for quantification of the neurofilament light chain in blood samples: ELISA, electrochemiluminescence immunoassay and Simoa. Clin Chem Lab Med. 2016;54(10):1655-61.

24. Gisslen M, Price RW, Andreasson U, Norgren N, Nilsson S, Hagberg L, et al. Plasma concentration of the neurofilament light protein (NFL) is a biomarker of CNS injury in HIV infection: a cross-sectional study. EBioMedicine. 2016;3:135-40.

25. Kametani F, Hasegawa M. Reconsideration of amyloid hypothesis and tau hypothesis in Alzheimer's disease. Front Neurosci. 2018;12:25.

26. Dasaro CR, Holden WL, Berman KD, Crane MA, Kaplan JR, Lucchini RG, et al. Cohort profile: World Trade Center health program general responder cohort. Int J Epidemiol. 2017;46(2):e9.

27. Clouston SA, Kotov R, Pietrzak RH, Luft BJ, Gonzalez A, Richards $M$, et al. Cognitive impairment among World Trade Center responders: long-term implications of re-experiencing the 9/11 terrorist attacks. Alzheimers Dement (Amst). 2016;4:67-75.

28. Clouston SAP, Diminich ED, Kotov R, Pietrzak RH, Richards M, Spiro A 3rd, et al. Incidence of mild cognitive impairment in World Trade Center responders: long-term consequences of re-experiencing the events on 9/11/2001. Alzheimers Dement (Amst). 2019;11:628-36.

29. Fandos N, Pérez-Grijalba V, Pesini P, Olmos S, Bossa M, Villemagne VL, et al. Plasma amyloid $\beta$ 42/40 ratios as biomarkers for amyloid $\beta$ cerebral deposition in cognitively normal individuals. Alzheimers Dement (Amst). 2017;8:179-87.

30. Nasreddine ZS, Phillips NA, Bedirian V, Charbonneau S, Whitehead V, Collin I, et al. The Montreal Cognitive Assessment, MoCA: a brief screening tool for mild cognitive impairment. J Am Geriatr Soc. 2005;53(4):695-9.
31. Elman JA, Jak AJ, Panizzon MS, Tu XM, Chen T, Reynolds CA, et al. Underdiagnosis of mild cognitive impairment: a consequence of ignoring practice effects. Alzheimers Dement (Amst). 2018;10: 372-81.

32. Carson N, Leach L, Murphy KJ. A re-examination of Montreal Cognitive Assessment (MoCA) cutoff scores. Int J Geriatr Psychiatry. 2018;33(2):379-88.

33. Skrondal A, Rabe-Hesketh S. Generalized latent variable modeling: multilevel, longitudinal, and structural equation models. Boca Raton: CRC; 2004.

34. Gardner W, Mulvey EP, Shaw EC. Regression analyses of counts and rates: Poisson, overdispersed Poisson, and negative binomial models. Psychol Bull. 1995;118(3):392-404.

35. McNutt LA, Wu C, Xue X, Hafner JP. Estimating the relative risk in cohort studies and clinical trials of common outcomes. Am J Epidemiol. 2003;157(10): 940-3.

36. Liu M, Paranjpe MD, Zhou X, Duy PQ, Goyal MS, Benzinger TLS, et al. Sex modulates the ApoE epsilon 4 effect on brain tau deposition measured by (18)F-AV-1451 PET in individuals with mild cognitive impairment. Theranostics. 2019;9(17): 4959-70.

37. Hilal S, Wolters FJ, Verbeek MM, Vanderstichele $\mathrm{H}$, Kamran Ikram M, Stoops E, et al. Plasma amyloid- $\beta$ levels, cerebral atrophy and risk of dementia: a population-based study. Alzheimers Res Ther. 2018;10:63.

38. Vergallo A, Mégret L, Lista S, Cavedo E, Zetterberg $\mathrm{H}$, Blennow $\mathrm{K}$, et al. Plasma amyloid $\beta$ 40/42 ratio predicts cerebral amyloidosis in cognitively normal individuals at risk for Alzheimer's disease. Alzheimer's Dement. 2019;15(6):764-75.

39. Mann DM, Brown SM, Owen F, Baba M, Iwatsubo T. Amyloid beta protein (A beta) deposition in dementia with Lewy bodies: predominance of $\mathrm{A}$ beta 42(43) and paucity of A beta 40 compared with sporadic Alzheimer's disease. Neuropathol Appl Neurobiol. 1998;24(3):187-94.

40. Dickson TC, Vickers JC. The morphological phenotype of beta-amyloid plaques and associated neuritic changes in Alzheimer's disease. Neuroscience. 2001;105(1):99-107.

41. Selden N, Mesulam MM, Geula C. Human striatum: the distribution of neurofibrillary tangles in Alzheimer's disease. Brain Res. 1994;648(2):327-31.

42. Ly JV, Donnan GA, Villemagne VL, Zavala JA, Ma H, $\mathrm{O}^{\prime}$ Keefe $\mathrm{G}$, et al. 11C-PIB binding is increased in 
patients with cerebral amyloid angiopathy-related hemorrhage. Neurology. 2010;74(6):487-93.

43. Cho H, Choi JY, Hwang MS, Kim YJ, Lee HM, Lee HS, et al. In vivo cortical spreading pattern of tau and amyloid in the Alzheimer disease spectrum. Ann Neurol. 2016;80(2):247-58.

44. Clouston SAP, Deri Y, Diminich E, Kew R, Kotov R, Stewart C, et al. Posttraumatic stress disorder and total amyloid burden and amyloid-beta $42 / 40$ ratios in plasma: results from a pilot study of World Trade Center responders. Alzheimers Dement (Amst). 2019;11:216-20.

45. Mielke $\mathrm{MM}$, Hagen $\mathrm{CE}, \mathrm{Xu} \mathrm{J}$, Chai $\mathrm{X}$, Vemuri $\mathrm{P}$, Lowe VJ, et al. Plasma phospho-tau181 increases with Alzheimer's disease clinical severity and is associated with tau- and amyloid-positron emission tomography. Alzheimers Dement. 2018;14(8): 989-97.
46. Clouston SAP, Deri Y, Horton M, Tang C, Diminich E, DeLorenzo C, et al. Reduced cortical thickness in World Trade Center responders with cognitive impairment. Manuscript submitted for publication (copy on file with author).

47. Clouston S, Diminich E, Kranidis A, Kotov R, Kuan $\mathrm{PF}$, Bromet E, et al. Posttraumatic stress-related cognitive and physical impairment: clinical characterization of a novel disorder. Manuscript submitted for publication (copy on file with author).

48. Crary JF, Trojanowski JQ, Schneider JA, Abisambra JF, Abner EL, Alafuzoff I, et al. Primary age-related tauopathy (PART): a common pathology associated with human aging. Acta Neuropathol. 2014;128(6): 755-66.

49. Jack CR Jr, Knopman DS, Chetelat G, Dickson D, Fagan AM, Frisoni GB, et al. Suspected non-Alzheimer disease pathophysiology-concept and controversy. Nat Rev Neurol. 2016;12(2):117-24. 